\title{
Espina de pescado alojada en la glándula tiroides
}

\author{
Fishbone lodged in the thyroid gland
}

\author{
Gabriela Morales $\mathbf{M}^{1}$, Paula De Las Heras F1․ Miguel Toledano T², Elisa Gil-Carcedo S1.
}

\begin{abstract}
RESUMEN
Los cuerpos extraños alojados en la vía aéreo-digestiva superior son un motivo de consulta frecuente para el otorrinolaringólogo. Uno de los más frecuentes son las espinas de pescado. En raras ocasiones, éstas migran hacia tejidos adyacentes y generan complicaciones importantes. Presentamos el caso de una paciente que tras la ingesta de pescado manifiesta sensación de cuerpo extraño faríngeo, odinofagia y dolor látero cervical derecho. Dada la ausencia de hallazgos a la exploración física y laringoscópica se realiza una tomografía computarizada cervical, visualizando un cuerpo extraño alojado en la glándula tiroidea. Se interviene mediante cervicotomía media, realizando la extracción de la espina sin complicaciones. El diagnóstico precoz y manejo adecuado es determinante para prevenir complicaciones en estos casos.
\end{abstract}

Palabras clave: Cuerpo extraño, espina de pescado, glándula tiroides, migración.

\begin{abstract}
Foreign bodies in the aerodigestive tract are commonly confronted in otolaryngology practice. The most frequent are the fish bones. In rare cases they can move and cause numerous complications. We report the case of a patient that manifest the sensation of pharyngeal foreign body, after fish ingestion. She also referred odynophagia and right cervical pain. Physical and laryngoscopic examination were normal. A cervical CT was performed, where a foreign body lodged in the thyroid gland was visualized, median cervicotomy was done and the foreign body was extracted. Early diagnosis and adequate management are crucial to prevent complications in these cases.
\end{abstract}

Key words: Foreign body, fish bone, thyroid gland, migration.

\footnotetext{
1 Servicio de Otorrinolaringología y Patología Cervicofacial. Hospital Universitario Río Hortega. Valladolid, España.

2 Servicio Cirugía General y del Aparato Digestivo. Hospital Universitario Río Hortega. Valladolid, España.

Los autores declaran no tener conflictos de interés.

Recibido el 16 de agosto de 2019. Aceptado el 8 de septiembre de 2019.
} 


\section{CASO CLÍNICO}

Mujer de 58 años que acude al servicio de urgencias por presentar sensación de cuerpo extraño faríngeo y odinofagia que empeoraba con la deglución, asociado a dolor látero cervical que se inició tras la ingesta de pescado (cabracho), el dolor se atenuaba parcialmente con antiinflamatorios. No presentó disfagia, disnea 0 fiebre. A la exploración física, la orofaringe era normal sin evidenciar a la inspección ni a la palpación cuerpos extraños; la palpación cervical no presentaba aumento de volumen ni signos inflamatorios, aunque refería dolor a la palpación del lóbulo tiroideo derecho. En la primera exploración laringoscópica no se evidencia cuerpo extraño en la vía aéreodigestiva superior (VADS), ni lesiones en la mucosa, ni retención salival en senos piriformes. Dada la persistencia de la clínica, se solicita una endoscopía digestiva alta, que fue normal. Se procede al alta de la paciente con observación domiciliaria y tratamiento conservador. A las 48 horas acude de nuevo al servicio de urgencias con aumento del dolor cervical y odinofagia de forma progresiva, sin nuevos síntomas. La exploración con laringoscopía es nuevamente normal. Se decide ingreso para tratamiento intravenoso con antiinflamatorios y antibiótico. Se solicita una tomografía computarizada (TC) cervical en donde se visualiza una imagen compatible con cuerpo extraño lineal, en las partes blandas de la región caudal de la hipofaringe derecha, en estrecha relación con el lóbulo tiroideo ipsilateral y adyacente a la vena yugular interna (VYI) (Figura 1).

Ante estos hallazgos realizamos una cervicotomía media exploratoria (incisión de Kocher) y se extrae cuerpo extraño localizado en el lóbulo tiroideo derecho (Figura 2), sin evidenciar hematoma, absceso cervical, perforación esofágica 0 alguna otra complicación. En vista al buen estado de la glándula tiroidea, se decide no realizar hemitiroidectomía. La paciente permanece hospitalizada en régimen cero durante 3 días manteniendo antibioterapia intravenosa, evolucionando de forma favorable.

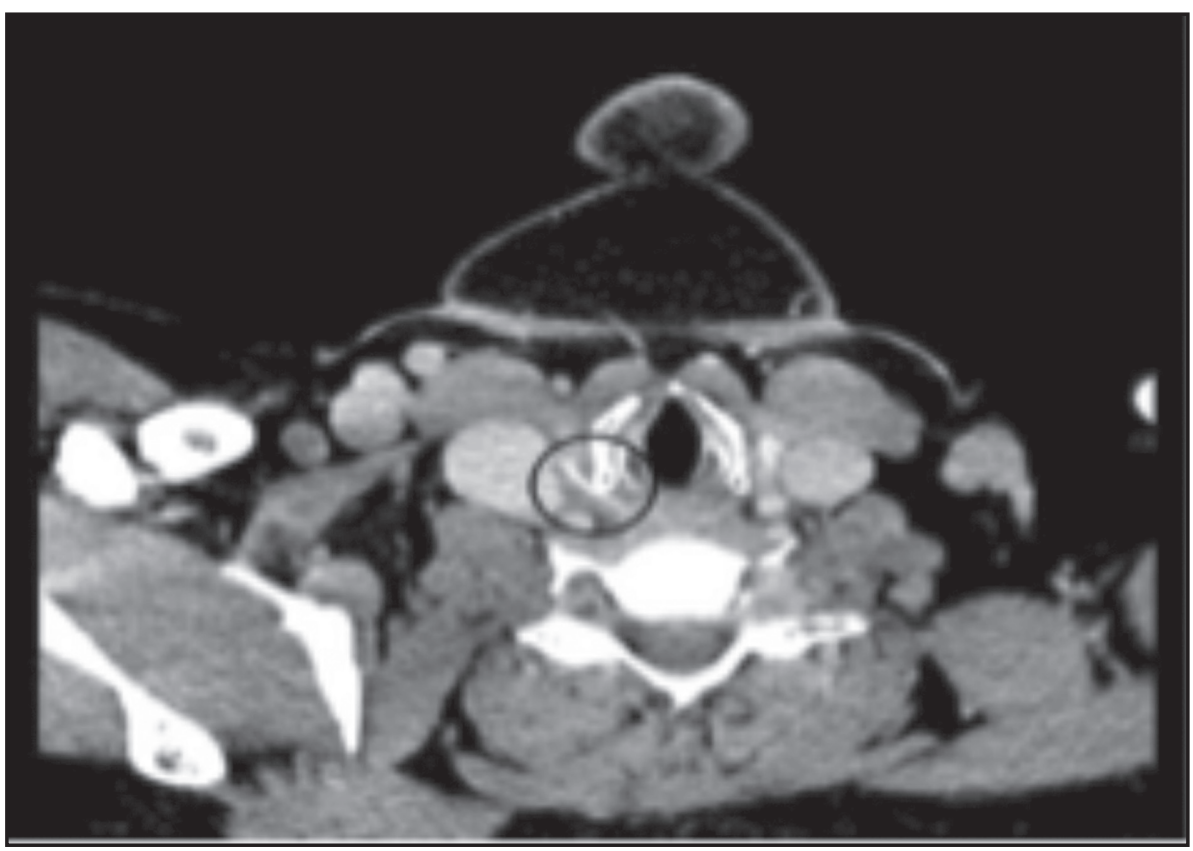

Figura 1. Tomografía computarizada cervical axial. Se observa cuerpo extraño lineal radiopaco, adyacente a VYI derecha en claro contacto con glándula tiroidea (círculo). 


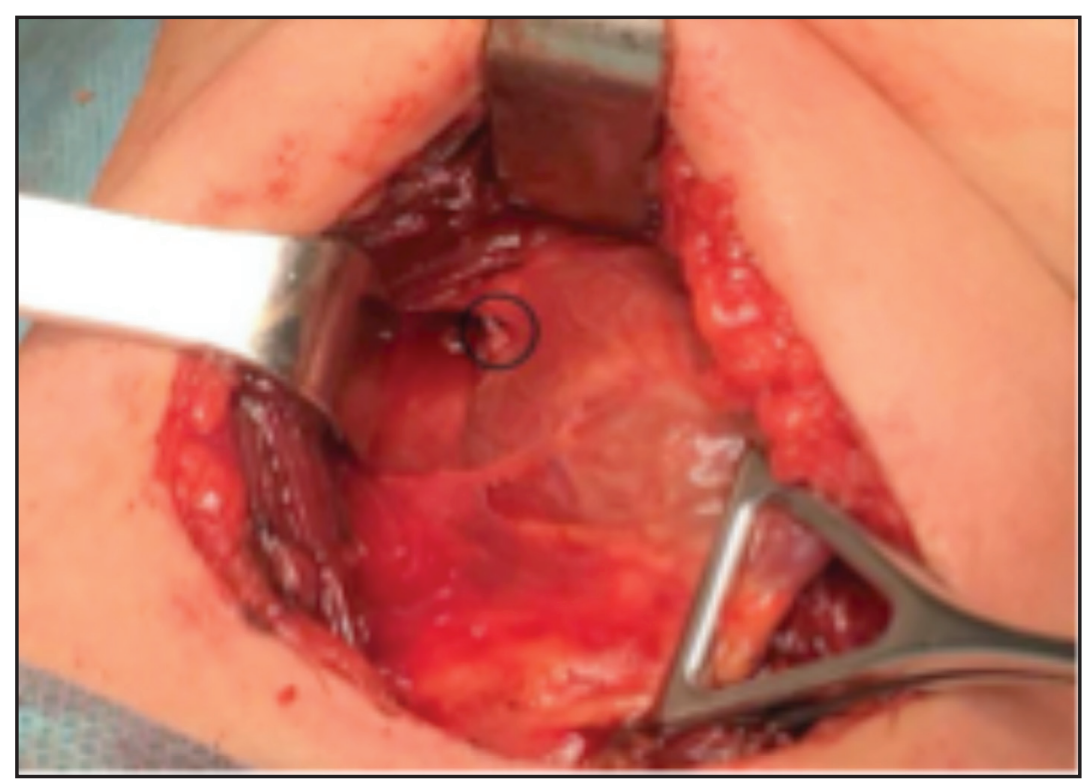

Figura 2. Cervicotomía media. Se identifica lóbulo tiroideo derecho con cuerpo extraño a nivel del polo superior del lóbulo tiroideo (círculo).

\section{DISCUSIÓN}

Los cuerpos extraños en las VADS son un motivo de consulta común para el otorrinolaringólogo ${ }^{1}$. Uno de los cuerpos extraños más observados en faringe y esófago cervical es la espina de pescado. Aproximadamente $80 \%-90 \%$ de los cuerpos extraños ingeridos pasan de forma espontánea sin dificultades, mientras que 10\%-20\% requieren extracción endoscópica y aproximadamente 1\% intervención quirúrgica para su extracción $n^{2}$. A menudo las espinas de pescado se anclan en las amígdalas palatinas 0 en la base de la lengua y son fáciles de extraer ${ }^{3}$. Si no se extraen, existe el riesgo que dañen la mucosa y ocasionen diversas complicaciones ${ }^{4}$, incluyendo abscesos cervicales profundos, mediastinitis, perforación esofágica y paresia de cuerda vocal, entre otras ${ }^{3}$. Las espinas de pescado localizadas en el exterior de la faringe, laringe y esófago son relativamente inusuales y es aún más raro que se alojen en la glándula tiroides ${ }^{4}$. El mecanismo por el cual los cuerpos extraños pasan a través del tejido blando se debe a los movimientos de los músculos del cuello, combinados con la peristalsis esofágica ${ }^{5}$. En ocasiones el diagnóstico se retrasa debido a que las manifestaciones clínicas suelen ser inespecíficas. Los síntomas más comunes son: sensación de cuerpo extraño, odinofagia, disfagia, dolor y aumento de volumen cervical ${ }^{2}$. Dentro de las pruebas complementarias que se pueden realizar están la radiografía cervical simple, cuya sensibilidad y especificidad para detectar espinas de pescado es de $39 \%$ y $72 \%$, respectivamente 6 . La ecografía no siempre es útil en estos casos, pero tiene el beneficio de ser no invasiva y fácil de realizar ${ }^{4}$. La TC tiene alta sensibilidad (90\%-100\%) y especificidad (93,7\%$100 \%$ ), con un VPP del $75 \%$ y un VPN del $97 \%{ }^{2}$. Esta prueba ayuda a determinar las características del cuerpo extraño, su relación con las estructuras que lo circundan y la extensión de su afectación, facilitando así su localización, para su posterior extracción. Es la prueba de imagen de elección en situaciones de alta sospecha de presencia de cuerpo extraño que no se logre valorar a la exploración endoscópica ni con otras pruebas de imagen 0 cuando existan signos de una complicación asociada. El manejo inicial incluye la evaluación de la vía respiratoria para identificar aquellos en riesgo de aspiración². En los casos en los que no se encuentre el cuerpo extraño durante la laringoscopía o endoscopía digestiva superior, 
se debe sospechar que la espina haya podido migrar al tejido blando cervical. En estos casos se deben realizar pruebas de imagen para conocer la posición exacta del cuerpo extraño o las posibles complicaciones asociadas e indicar un tratamiento quirúrgico². Cuando la espina de pescado migra a la glándula tiroides, dependiendo de la afectación de ésta y de las complicaciones asociadas, puede ser necesario realizar una hemitiroidectomía ${ }^{4}$.

\section{CONCLUSIÓN}

Las espinas de pescado en VADS son una causa común en la consulta de ORL. La mayoría

\section{BIBLIOGRAFÍA}

1. Shergill GS, Nayak DR, Dora A, Shergill AK. Migrating foreign bodies in the upper aerodigestive tract: a surgical challenge. BMJ Case Rep 2015, bcr2015210326. doi: 10.1136/ bcr-2015-210326.

2. KIM HU. Oroesophageal fish bone foreign body. Clin Endosc 2016; 49: 318-26.

3. Vallamkondu V, Carlile S, Shakeel M, Ah-See KW. Neck abscess and vocal cord paresis: delayed complications of a self-extruded long fishbone stuck in throat. BMJ Case Rep 2013. doi:10.1136/bcr-2013-201832. de las veces avanzan al tracto digestivo y la situación se resuelve de forma espontánea. En ocasiones su extracción suele ser dificultosa. La posibilidad que perfore y migre hacia los tejidos blandos del cuello debe sospecharse siempre ante la persistencia de los síntomas. Aunque esta situación es poco frecuente, se debe realizar un diagnóstico temprano y tratamiento adecuado para prevenir las complicaciones asociadas. En los casos de migración al tiroides se debe determinar el grado de afectación del tejido glandular y tejido circundante, incluyendo el esófago cervical, para decidir el tratamiento adecuado que puede incluir la hemitiroidectomía.
4. Wu E, Huang L, Zhou Y, Zhu X. Migratory fish bone in the thyroid gland: Case Report and Literature Review. Case Rep Med 2018. doi:10.1155/2018/7345723.

5. Adil T, Btissam B, Othmane B, Hassane N, Youssef R, ET AL. A Foreign Body in the Pharynx Migrating through the Internal Jugular Vein: Case Report of Unusual Complication. Otolaryngology 2014; 4: 1000172. doi:10.4172/2161$119 X .1000172$.

6. Johari HH, Khaw BL, Yusof Z \& Mohamad I. Migrating fish bone piercing the common carotid artery, thyroid gland and causing deep neck abscess. World J Clin Cases 2016; 4: 375-9.

Correspondencia: Gabriela Morales M. 